\title{
Custom Modification of AFM Tips for Fast, High Force Resolution Single-Molecule Force Spectroscopy.
}

\author{
Aric W. Sanders ${ }^{1}$, Jaevyn K. Faulk ${ }^{2}$, Devin T. Edwards ${ }^{2}$ and Thomas T. Perkins ${ }^{2}$ \\ 1. National Institute of Standards and Technology, Boulder, Colorado, USA. \\ 2. JILA, National Institute of Standards and Technology and University of Colorado, Boulder, Colorado \\ 80309, United States
}

In addition to providing the ability to image on the nanoscale, atomic force microscopy (AFM) has the ability to measure small ( $\mathrm{pN}$ ) forces. This ability has led to new insights into conformational changes in biological molecules; in particular, single-molecule force spectroscopy (SMFS) is a powerful tool to investigate folding in proteins. Ideally, one could observe folding in proteins at time scales in the microsecond range with both short-term force precision and long-term force stability. Recent work [1] has shown that to minimize force drift, one must use a soft AFM cantilever due to instrumental noise. Such soft, long cantilevers have poor temporal resolution. When high temporal resolution is required, one choses a shorter cantilever, which has a higher spring constant $\left(k \propto L^{-3}\right)$ and hence increased force drift. Recently, it has been shown that by modifying a commercially available cantilever with focused ion beam (FIB) milling, short AFM cantilevers $(\mathrm{L} \sim 40 \mu \mathrm{m})$ can be softened to reduce force drift, without sacrificing temporal resolution. The resulting cantilevers offer state-of-the-art force stability and precision, with temporal resolution $\sim 70 \mu \mathrm{s}$ [2]. To further improve the performance of modified AFM cantilevers, FIB modification strategies need to be developed to extend the technique to ultra-small cantilevers $(\mathrm{L} \sim 9 \mu \mathrm{m})$, which offer temporal resolution $\sim 1 \mu \mathrm{s}$ [3].We present several modification strategies, each of which decrease the stiffness of the resultant cantilevers while retaining fast response times. Additionally, we explore techniques that improve the yield of the process.

Our basic process of AFM cantilever modification relies on decreasing the stiffness of a commercially available cantilever, such as a Biolever Mini or Bio Lever Fast [4]. The reduction of stiffness is achieved primarily through the removal of material on the cantilever through Ga-ion-based FIB milling. We are also exploring He-ion milling. This milling is either used to thin the full cantilever (Figure 1) or to remove a rectangle of material and then to thin the remaining supports (Figure 2). In addition to lowering $\mathrm{k}$, removing a cantilever's gold coating has been shown to reduce force drift [5]. This is achieved by protecting an area using electron beam induced deposition of a suitable etch mask, and then wet etching most of the gold away leaving a reflective surface to measure the cantilever, or FIB-milling of the gold.

These methods have successfully produced FIB-modified, ultra-short cantilevers with pN integrated force noise $(1-350 \mathrm{~Hz})$ and $\mu \mathrm{s}$ temporal resolution. Yet, the yield of the process is limited by the tendency of the cantilevers to bend while being modified. This bending can cause issues with milling. More importantly, if the cantilevers remain highly bent, they are not measurable by the optical deflection laser. The bending of cantilevers is seen to be a function of both electron and ion exposure and several strategies to minimize both have been developed. (see Figure 2). For sufficiently long cantilevers ( $\mathrm{L} \geq 40$ $\mu \mathrm{m})$, exposure to electrons causes little to no bending, whereas ultra-small cantilevers bend quickly. We present a reproducible protocol to FIB-modify cantilevers of varying sizes without bending. 


\section{References:}

[1] Churnside, A. B. and T. T. Perkins FEBS Letters 588 (2014), p 3621-3630.

[2] Bull, M. S., et al. ACS Nano 8 (2014), p 4984-4995.

[3] Rico et al., Science 342 (2013), p 741-743

[4] Certain commercial equipment, instruments, or materials are identified in this paper to foster understanding. Such identification does not imply recommendation or endorsement by the National Institute of Standards and Technology, nor does it imply that the materials or equipment identified are necessarily the best available for the purpose

[5] Churnside, A. B., et al. Nano Letters 12 (2012), p 3557-3561.

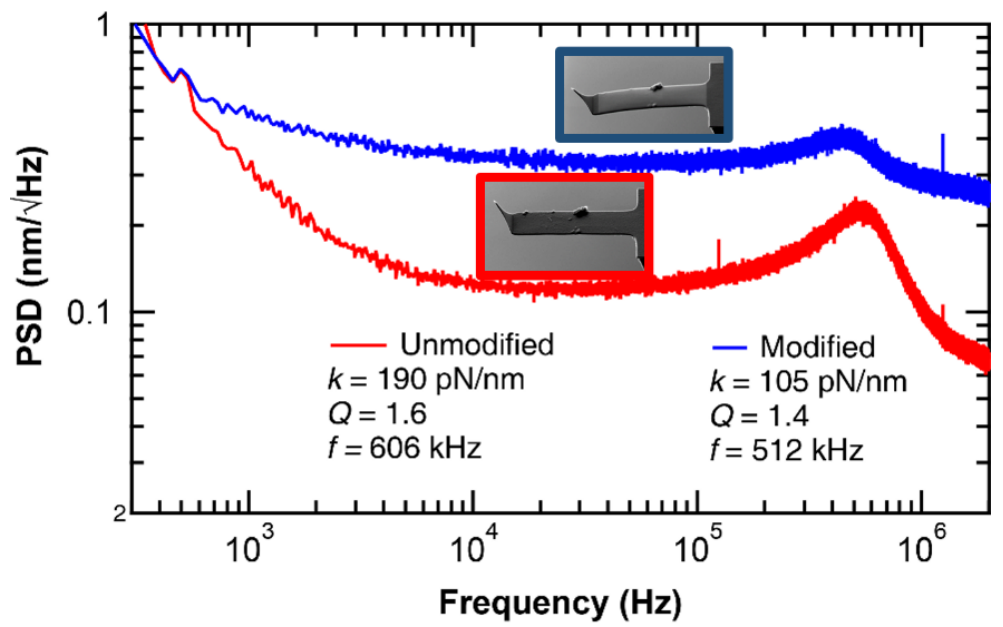

Figure 1. A modified cantilever, with thermal noise curve. This cantilever, a Biolever fast, has been thinned from the bottom, decreasing the spring constant from $187 \mathrm{pN} / \mathrm{nm}$ to $105 \mathrm{pN} / \mathrm{nm}$. In this case the modified cantilever has higher force noise, an occasional undesirable side effect of modification.

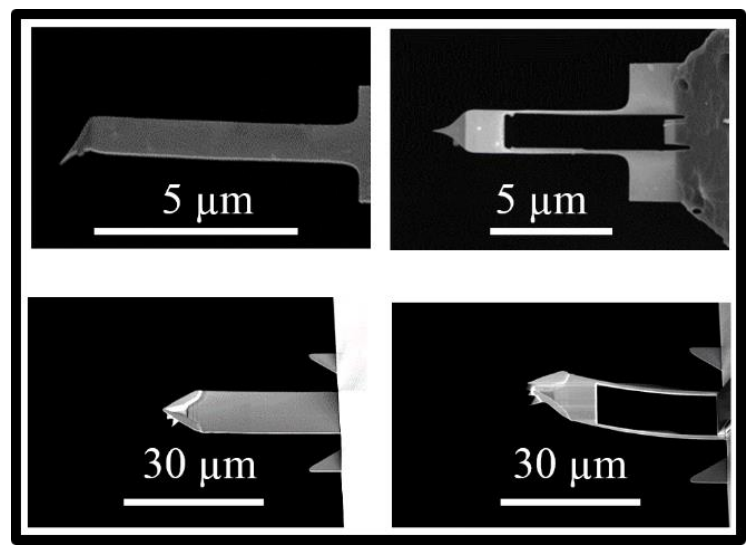

Figure 2. Modification of AFM cantilevers to reduce stiffness by removal of a central section. Top left, unmodified ultra-small cantilever. Top right, cantilever after modification imaged normal to cantilever. Bottom left, an unmodified small cantilever. Bottom right, a tilted image of the modified cantilever showing a large degree of bending. 